\title{
270-km Ultralong Raman Fiber Laser
}

\author{
S. K. Turitsyn, ${ }^{1}$ J. D. Ania-Castañón, ${ }^{1,2}$ S. A. Babin, ${ }^{3}$ V. Karalekas, ${ }^{1}$ P. Harper, ${ }^{1}$ D. Churkin, ${ }^{3}$ S. I. Kablukov, ${ }^{3}$ \\ A.E. El-Taher, ${ }^{1}$ E. V. Podivilov, ${ }^{3}$ and V. K. Mezentsev ${ }^{1}$ \\ ${ }^{1}$ Photonics Research Group, Aston University, Birmingham, B4 7ET, United Kingdom \\ ${ }^{2}$ Instituto de Óptica, CSIC, C/ Serrano 121, Madrid 28006, Spain \\ ${ }^{3}$ Institute of Automation and Electrometry, SB RAS, Novosibirsk 630090, Russia
}

(Received 1 June 2009; published 21 September 2009)

\begin{abstract}
We analyze the physical mechanisms limiting optical fiber resonator length and report on the longest ever laser cavity, reaching $270 \mathrm{~km}$, which shows a clearly resolvable mode structure with a width of $\sim 120 \mathrm{~Hz}$ and peak separation of $\sim 380 \mathrm{~Hz}$ in the radio-frequency spectrum.
\end{abstract}

DOI: 10.1103/PhysRevLett.103.133901

Lasers are usually considered simply as sources of coherent light. However, an ultralong laser cavity implemented in optical fiber can also be seen as a unique transmission medium leading to a radical new outlook on information transmission and secure communications. Despite extraordinary advances in laser science, only recently have the fundamental limits of laser cavity length become an area of exploration. Here we study physical mechanisms that restrict boundless increase of a fiber laser cavity and present a new system design that allows us to approach for the first time the theoretical limits set by these physical effects.

Increases by several orders of magnitude in resonator length compared to solid state or gas lasers can be achieved by using an optical fiber waveguide and the Raman scattering effect. Raman fiber lasers exploit stimulated Raman scattering to overcome fiber loss and create an effective gain medium at the shifted Stokes wavelengths [1] using the transmission fiber itself as the laser resonator [2]. In [36 ] it was demonstrated that cavity length could be extended to tens of km, allowing novel applications of lasers such as quasilossless optical signal transmission. This is of particular interest for soliton-based systems and signal processing $[7,8]$, as well as for the design and practical implementation of photonic devices based on the mathematical theory of integrable nonlinear systems [9-11]. An ideal transmission medium could be defined as a physical link between transmitter and receiver that does not corrupt or distort the signal in any way, within the whole spectral transmission band. In practice, unfortunately, this situation of true transparency has never been approachable but for the shortest possible transmission distances, as optical gain or loss is normally frequency dependent. Ultralong lasers have allowed spatio-spectral transparency at long distances over a broad bandwidth even with only single-frequency laser pumps, thanks to the extended signal gain bandwidth provided by the superposition of a pump and cascading Stokes wave $[5,6]$. Recent results showing simultaneous transparency over $20 \mathrm{~nm}$ for standard fiber links of $20 \mathrm{~km}$ length [6], have opened the gate to a series of novel applications, from signal processing devices in which different
PACS numbers: 42.55.Ye, 42.60.By, 42.79.Sz, 42.81.Wg

channels can be made to nonlinearly interact with each other in nondissipative conditions, to quantum communication systems relying on continuous quantum variables, for which these transparent links might provide an ideal medium to perform experiments on self-phase modulation squeezing of quantum optical solitons [12]. Quantum communications with transmission of few photons are another option for which our transparent links seem to be suitable in principle, although this would, of course, require an extremely precise stabilization of the cavity characteristics, as well as of the energy of the Stokes wave trapped in the cavity. In addition, ultralong laser cavities can be exploited in fundamentally new practical approaches for optical information transmission and secure communications. For instance, the concept of nonquantum secure key distribution based on establishing laser oscillations between the sender and receiver has been recently proposed in $[13,14]$.

Here we depart from previous studies of fiber lasers and drastically increase cavity length in order to explore the fundamental limits of laser operation. This endeavor has resulted in the discovery of new physics underlying light generation regimes, different from classical laser operation, which are the main subject of this study. In particular, the huge number of weakly interacting longitudinal modes present in the cavity results in interesting properties that resemble the random behavior associated with the socalled weak wave turbulence [15-17]. Another important physical mechanism is distributed Rayleigh backscattering [18]. We demonstrate here that in the ultralong laser under study both effects influence the final mode structure to different degrees depending on power, and can result in "modeless" spectra at some power levels.

Our experimental configuration is schematically represented in Fig. 1. Using this configuration, a single-stage scheme pumped at $\sim 1450 \mathrm{~nm}$ and laser operation at Stokes wavelength of $\sim 1550 \mathrm{~nm}$, we will demonstrate that a laser resonator can be extended beyond $270 \mathrm{~km}$ while retaining a resolvable mode structure at low power, confirming the formation of an ultralong standing electromagnetic wave between the cavity mirrors. In order to achieve 


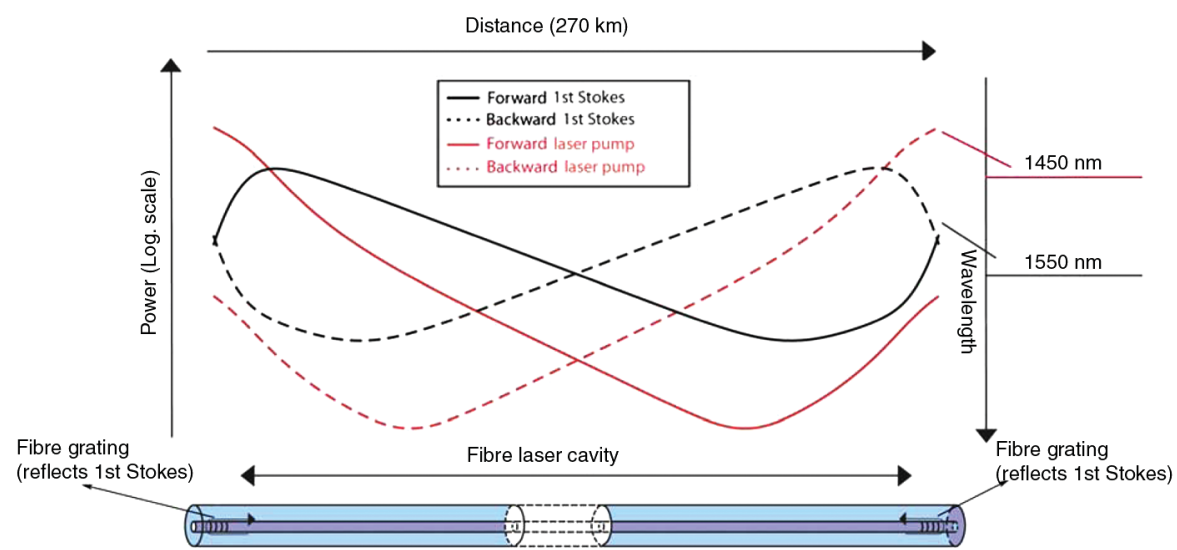

FIG. 1 (color). Schematic of the ultralong Raman fiber laser design and calculated optical powers distribution.

this, we take advantage of the reduced attenuation in the telecommunication window of the optical fiber, which includes both the wavelength of the pumping lasers and those of the generated laser radiation. Note that this configuration can also be exploited to provide quasilossless optical signal transmission in the region of $1650-1675 \mathrm{~nm}$ which is of interest too in optical communications. Fiber Bragg gratings (FBGs) positioned at the ends of the transmission fiber play the role of mirrors for the generated laser radiation at $1550 \mathrm{~nm}$.

Note that Rayleigh backscattering (RBS) has a serious impact on the longitudinal distribution of radiation intensity, making it nonmonotonic. This effect is particularly pronounced in the case of the initial pumps, providing for their growth at the opposite end of the cavity (see Fig. 1) through coupling of a part of the elastically scattered forward-propagating radiation into the backwardpropagating direction and vice-versa. Except for the operating wavelength, the experimental scheme is similar to that in our previous studies of ultralong Raman fiber lasers (URFLs) with shorter cavity length, $L<84 \mathrm{~km}[19,20]$. Let us repeat the most important details: Corning single mode fiber (SMF) spliced spans with total length ranging from 105 to $270 \mathrm{~km}$ and highly reflective $(R \sim 99 \%$ at $\sim 1550 \mathrm{~nm}$ ) fiber Bragg gratings (FBG) with a bandwidth of $\sim 1 \mathrm{~nm}$ are used. Two pump lasers operating at $\sim 1450 \mathrm{~nm}$ are coupled into the fiber ends with FBGs via wavelength-division multiplexing (1450/1550) couplers. When the pump power reaches the threshold, lasing at $1550 \mathrm{~nm}$ starts. Intracavity power of the Raman laser is monitored via $1 \%$ port of the 1:99 splitter inserted near the FBG. In addition, rf and optical spectra are measured using electrical and optical spectrum analyzers, correspondingly.

Using this setup we monitored beating peaks in the radio-frequency spectra with $100-200 \mathrm{~Hz}$ width and $\sim 1$, $\sim 0.6, \sim 0.5$ and $\sim 0.38 \mathrm{kHz}$ spacing for 105, 165, 208 and $270 \mathrm{~km}$ lasers, respectively. In Fig. 2 the new data are shown together with previous experimental points for $L<$ $84 \mathrm{~km}[19,20]$ to stress the difference from previous measurements. Our predictions in [20] concerning achievable cavity length did not take into account physical limitations due to distributed Rayleigh backscattering. As we will see, the RBS effect is critical for further lengthening the cavity. The observed spectral separation of the longitudinal modes clearly follows the classical formula $\Delta v=c / 2 n L$ (solid curve), where $n$ is the refractive index of the fiber core, $c$ the speed of light and $L$ the cavity length. The fiber Bragg gratings at both ends of the cavity trap photons within their bandwidth $B$. Thus, the number of the longitudinal modes generated in a resonator $\approx B / \Delta \nu \propto L$ is proportional to the length of the cavity. Therefore, the number of modes in an URFL cavity is extremely large - up to hundreds of millions for a typical value of $B \sim 100 \mathrm{GHz}$ and a spectral mode spacing $\Delta \nu$ of $1 \mathrm{kHz}$ at length $L \sim 100 \mathrm{~km}$. We would like to stress that the amazing fact here is not that the mode spacing is in full agreement with this fundamental laser law, but that we still have an observable mode structure for such extremely long cavities. This poses questions concerning the physical mechanisms underlying

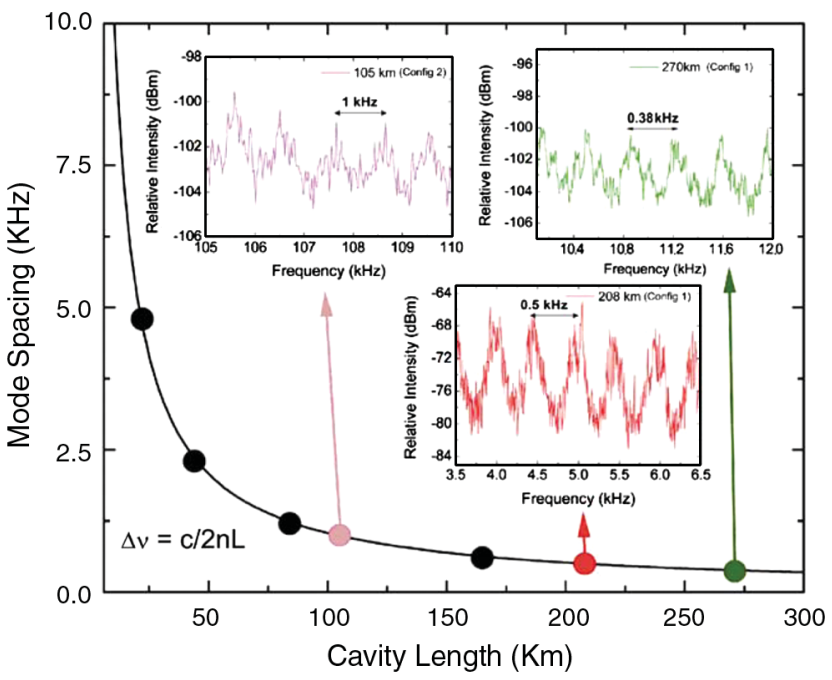

FIG. 2 (color). Experimental demonstration of the resolvable mode structure with the spectral spacing following the classical laser law $\Delta \nu=c / 2 n L$. Insets: intensity beating spectra for 105 , 208, and $270 \mathrm{~km}$ lasers. 
the buildup of radiation in such an unusually long FabryPerot resonator.

Next we examined the impact of fiber nonlinearity (and intracavity power) on the properties of the generated radiation, first of all, on its mode structure. Note that while rf peak width $\delta$ is almost independent of cavity length, it grows nearly linearly with increasing Stokes wave intensity $\delta \infty I$ as shown in Fig. 3. This clearly indicates that intracavity nonlinear effects play an important role in URFL operation. When the extrapolated width $\delta(I)$ reaches the value of the mode spacing $\Delta \nu$ (corresponding values for each cavity length are shown in Fig. 3 by horizontal lines), the mode structure is "washed out" because of the turbulentlike phase shifts induced by nonlinear interactions of multiple waves with different frequencies. Thus, the determined maximum power with a resolved mode structure is inversely proportional to the length. For $L=270 \mathrm{~km}$ it amounts to a value as low as $I_{\max } \sim 15 \mathrm{~mW}$, and as a result a clear mode structure is observable only at much lower powers.

As we have shown, by operating close enough to the threshold limit, we have been able to clearly resolve cavity modes up $270 \mathrm{~km}$. Even though we have been able to detect periodicity resembling a modal structure up to $303 \mathrm{~km}$, our accuracy at this length was below the levels required for the appropriate characterization of $\Delta \nu$. In addition, the laser length with a resolvable mode structure is limited not solely by nonlinear interactions between the modes, but also by Rayleigh backscattering, which becomes increasingly important with longer resonator lengths. Part of the scattered light is recaptured by the fiber waveguide leading to distributed reflection with an effective integral coefficient per unit length (at $1550 \mathrm{~nm}) \varepsilon_{1550 \sim} 4.5 \times 10^{-5} \mathrm{~km}^{-1}$. Multiple random reflections of forward radiation inside the transmission fiber play the role of a distributed mirror. Because of the random character of such reflections, they diffuse the effective cavity length parameter forming multiple effective resonators of randomly varying length, leading to the overlapping of modes and creating modeless radiation. The impact of RBS on laser operation becomes critically important when its contribution to the balance of gain and loss in the cavity is compared to the effect of reflections from FBGs. Such a critical cavity length can be estimated by comparing the classical lasing threshold condition on the pumping waves power: $R \exp [-\alpha L+$ $\left.g_{R} \int_{0}^{L} P_{p}(z) d z\right]=1$ with a balance equation for a cavity formed by one FBG and an effective "distributed mirror" due to RBS: $\varepsilon_{1550} R \int_{0}^{L} d z \exp \left[-2 \alpha z+2 g_{R} \int_{0}^{z} P_{p}(s) d s\right]=$ 1 . Here $L$ is the cavity length, $R$ is FBG reflectivity, $P_{p}(z)$ is the pump power distribution, $g_{R}$ is the Raman gain coefficient, and $\alpha$ is the loss coefficient for the generated Raman waves. Without taking into account the effect of the RBS the lasing threshold power $P_{\mathrm{th}}^{\mathrm{FBG}}$ grows linearly with a cavity length $L$. At a certain resonator length, lasing threshold $P_{\text {th }}^{\mathrm{RBS}}$ determined by the RBS impact-meaning lasing in the cavity formed by the first FBG and the

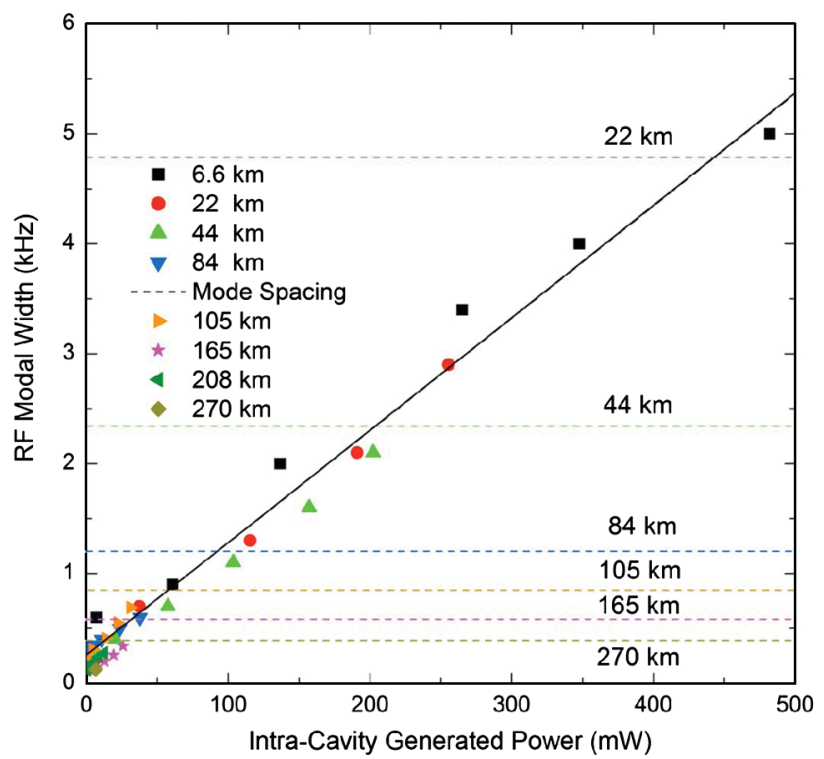

FIG. 3 (color). Mode beating peak width (FWHM) for 6.6., 22, 44, 84, 105, 165, 208, and $270 \mathrm{~km}$ lasers (differently marked points) and their linear fit. Corresponding mode spacing values are shown by horizontal lines.

distributed mirror (even without the second FBG)-becomes comparable to $P_{\mathrm{th}}^{\mathrm{FBG}}$ and the laser operation regime is changed with the corresponding diffusion of mode structure. Numerical modeling shows that for the symmetric configuration 1 the $P_{\text {th }}^{\mathrm{RB}}$ threshold approaches $P_{\text {th }}^{\mathrm{FBG}}$ level at cavity lengths $\approx 250 \mathrm{~km}$. Indeed, our experiments show that in cavities longer than $270 \mathrm{~km}$ (e.g., $303 \mathrm{~km}$ was tested), the impact of distributed Rayleigh backscattering appears to be important. At $L=303 \mathrm{~km}$ the peak structure in the radio-frequency spectra is hardly resolvable, since RBS effect determines the lasing reducing the impact of the second FBG.

Let us now discuss an interesting interdisciplinary link between URFL and the theory of wave turbulence [15-17]. Generated laser power is distributed between multitudes of very small amplitude cavity modes. However, the resonator is not linear and as seen in Fig. 3 nonlinear interactions between the modes does occur. The spectra of generated radiation experience spectral broadening dependent on power. Thus, the nonlinear Kerr effect does affect the propagation of light in URFL and leads to nonlinear mixing of the cavity modes. Any particular interaction between resonator modes can be considered as a weak one-the properties of each wave are not changed substantially during a single interaction event. However, despite the small amplitudes of propagating waves the overall effect can be important because of the huge number of modes involved in the interactions. The efficiency of interactions depends on resonance conditions between the phases of any four participating waves-the so-called four-wave mixing (FWM) process. Even weak nonlinear interactions through the FWM process lead to a random energy transfer 
between waves and to enhancement of mode dephasing because of a continuum of involved modes propagating with significant group velocity dispersion. Note that other manifestations of the Kerr nonlinearity such as self-phase or cross-phase modulation effects might be important for overall optical field evolution, but they do not lead to the intermode phase difference as they change the mode phases synchronously. We stress that the modes' dynamics are randomized not by noise, but due to the FWM nonlinear process that engages a huge number of deterministic interactions of rapidly oscillating modes with different amplitudes and phases making time evolution of any particular cavity mode extremely stochastic. The situation is somewhat similar to a classical physical problem in which long term average properties of the system are determined by the random behavior of a huge number of weakly interacting waves (which could be of quite different physical origins) - weak wave turbulence [15-17]. This link between the research areas of wave turbulence and laser physics also offers an opportunity to study the properties of wave turbulence in an optical device (laser) that allows very precise measurements. Note that recently such a similarity was successfully used in studies of optical rogue waves [21].

In conclusion, we have demonstrated the resolvable longitudinal mode structure of the longest ever $(270 \mathrm{~km})$ cavity laser, with longitudinal mode radio-frequency peaks of $\sim 120 \mathrm{~Hz}$ width and $\sim 380 \mathrm{~Hz}$ spectral spacing. Our experiments show that mode structure resolvability depends both on the pumping wave power and cavity length, with transition to modeless spectra above some values. Broadening of the radio-frequency peaks increases with power and, thus, has a nonlinear nature. Ultralong lasers represent a new exciting field of research that combines diverse areas of science. The physical mechanisms underlying the operation of such lasers involve nontrivial nonlinear interactions of the resonator modes and are quite different from those in other types of lasers. Lasers with such a large number of cavity modes have never been studied before. Therefore, we believe that our results might open an entirely new research field closely linked with a range of other areas of physics such as nonlinear science, theory of disordered systems, wave turbulence, and others. In addition, the concept of an ultralong fiber laser offers a promising opportunity for the implementation of effectively lossless transmission links with an extremely broad spectral bandwidth. A very interesting new application of ultralong lasers, as a new type of classical key distribution system that might overcome the practical challenges faced by quantum key distribution systems, has been recently proposed in $[13,14]$. In addition to such interesting connections with many areas of fundamental science, we also anticipate that new applications and technologies will keep emerging from the study of the physics of ultralong fiber lasers.

The authors acknowledge support of the EPSRC, The Royal Society, the Nuffield Foundation, the British Council, the integration project of Siberian Branch of Russian
Academy of Sciences, the Russian Ministry of Science and Education, and Grant No. TEC2008-05791 from the Spanish Ministry of Science and Innovation.

[1] R. H. Stolen, E. P. Ippen, and A. R. Tynes, Appl. Phys. Lett. 20, 62 (1972).

[2] S. G. Grubb, T. Strasser, W. Y. Cheung, W. A. Reed, V. Mizrahi, T. Erdogan, P. J. Lemaire, A. M. Vengsarkar, and D. J. Digiovanni, in Proc. Optical Amplifiers and Their Applications 1995 (Optical Society of America, Washington, DC, 1995), pp. 197-199.

[3] J. D. Ania-Castañón, Opt. Express 12, 4372 (2004).

[4] J.D. Ania-Castañón, T. J. Ellingham, R. Ibbotson, X. Chen, L. Zhang, and S. K. Turitsyn, Phys. Rev. Lett. 96, 023902 (2006).

[5] T. J. Ellingham, J.D. Ania-Castañón, R. Ibbotson, X. Chen, L. Zhang, and S. K. Turitsyn, IEEE Photonics Technol. Lett. 18, 268 (2006).

[6] J. D. Ania-Castañón, V. Karalekas, P. Harper, and S. K. Turitsyn, Phys. Rev. Lett. 101, 123903 (2008).

[7] L.F. Mollenauer and J.P. Gordon, Solitons in Optical Fibers: Fundamentals and Applications (Academic Press, New York, 2006).

[8] A. Hasegawa, IEEE J. Sel. Top. Quantum Electron. 6, $1161(2000)$

[9] S. Novikov, S. V. Manakov, L. P. Pitaevskii, and V.E. Zakharov, Theory of Solitons. The Inverse Scattering Method (in Russian: Nauka, Moscow, 1980; in English: Plenum Press, New York, 1984).

[10] What is Integrability?, edited by V. E. Zakharov, Springer Series in Nonlinear Dynamics (Springer-Verlag, Berlin, 1991).

[11] A.C. Newell, Solitons in Mathematics and Physics (Society for Industrial and Applied Mathematics, Philadelphia, 1985).

[12] P.D. Drummond, R. M. Shelby, S. R. Friberg, and Y. Yamamoto, Nature (London) 365, 307 (1993).

[13] J. Scheuer and A. Yariv, Phys. Rev. Lett. 97, 140502 (2006).

[14] A. Zadok, J. Scheuer, J. Sendowski, and A. Yariv, Opt. Express 16, 16680 (2008).

[15] V.E. Zakharov, V.S. L'vov, and G.E. Falkovich, Kolmogorov Spectra of Turbulence I: Wave Turbulence (Springer-Verlag, Berlin, 1992).

[16] G.E. Falkovich, Introduction to Turbulence Theory, Lecture Notes on Turbulence and Coherent Structures in Fluids, Plasmas and Nonlinear Media Vol. 4 (World Scientific, Singapore, 2006).

[17] V. E. Zakharov, P. Guyenne, A. N. Pushkarev, and F. Dias, Physica (Amsterdam) 152153D, 573 (2001).

[18] Lord Rayleigh (J. W. Strutt), Philos. Mag. 47, 375 (1899).

[19] S. A. Babin, V. Karalekas, P. Harper, E. V. Podivilov, V. K. Mezentsev, J. D. Ania-Castañón, and S. K. Turitsyn, Opt. Lett. 32, 1135 (2007).

[20] S. A. Babin, V. Karalekas, E. V. Podivilov, V. K. Mezentsev, P. Harper, J.D. Ania-Castañón, and S. K. Turitsyn, Phys. Rev. A 77, 033803 (2008).

[21] D. R. Solli, C. Ropers, P. Koonath, and B. Jalali, Nature (London) 450, 1054 (2007). 